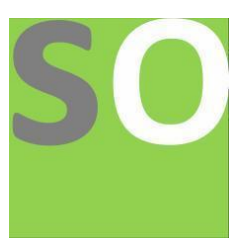

Article title: COVID-19 And SARS-COV-2 Infection And Virulence: Hypothesis I

Authors: H.Y. Lim Tung[1]

Affiliations: Nachbraht Biomedical Research Institute[1]

Orcid ids: 0000-0002-7112-7477[1]

Contact e-mail: hyltung2010@nacbrahtbiomedresins.net

License information: This work has been published open access under Creative Commons Attribution License http://creativecommons.org/licenses/by/4.0/, which permits unrestricted use, distribution, and reproduction in any medium, provided the original work is properly cited. Conditions, terms of use and publishing policy can be found at https://www.scienceopen.com/.

Preprint statement: This article is a preprint and has not been peer-reviewed, under consideration and submitted to ScienceOpen Preprints for open peer review.

DOI: 10.14293/S2199-1006.1.SOR-.PPXVKPX.v1

Preprint first posted online: 10 April 2020

Keywords: COVID-19, SARS-COV-2, Infectivity, Virulence, DP10000 Number 


\title{
COVID-19 and SARS-COV-2 INFECTION AND VIRULENCE: HYPOTHESIS I
}

\author{
H.Y. Lim Tung
}

Peptide and Protein Chemistry Research Laboratory, Nacbraht Biomedical Research Institute, 3164 21st Street, Suite 117, Astoria (NYC), New York 11106, USA.

Correspondence to:

H.Y. Lim Tung

Peptide and Protein Chemistry Research Laboratory

Nachbraht Biomedical Research Institute

316421 st Street, Suite 122

Astoria (NYC), NY 11106

USA

Tel: $646-500-1728$

E mail: hyltung2010@nacbrahtbiomedresins.net 


\begin{abstract}
.
SARS-COV-2 is the etiologic agent of COVID-19. There is currently no effective means of preventing infections by SARS-COV-2, except through restriction of population movement and contact. An understanding of the origin, evolution and biochemistry (molecular biology) of SARS-COV-2 is a prerequite to its control. There is no definitive answer as to the origin of SARS-COV-2. The evolution of SARS-COV-2 can be gleaned from a comparative study of its infectivity and virulence in different populations and environments. From an analysis of the infectivity and virulence of SARS-COV-2 in different parts of the world, it is submitted that there is no correlation between infectivity and virulence of SARS-COV-2. It is therefore hypothesized that SARS-COV-2 may be a rapidly mutating virus and identification of SARS-COV-2 strain(s) that has/have increased virulence is essential in the formulation of any effective vaccine or prophylactic that targets SARS-COV-2.
\end{abstract}


SARS-COV-2 is the etiologic agent of COVID-19 which is now considered a Pandemic [1-4]. Currently, the only effective means of avoiding morbidity due to infections by SARS-COV-2 is through population control and contact restriction which appear to be quite effective in some parts of the world and not others. However, the number of deaths in various populations and regions of the world are uniform (See below). Although the medical infrastructure and response strategy and protocol play an important role in mitigating illness and death as a result of SARS-COV-2 infection, the possibility that variability in the number of deaths following SARS-COV-2 may be due to SARS-COV-2 mutations cannot be ruled out.

As a first step in determining whether the virulence of SARS-COV-2 may be changing and different in various populations and regions because of mutation(s) in SARS-COV-2, the number of deaths per 10,000 individuals infected with SARS-COV-2 or DP10000 Number in a given population was calculated based on the available data from the World Heath Organization, the Center for Disease Control and Prevention and from local Departments of Health Services. Figure 1, shows that in the United States, there was an exponential growth in the number of individuals infected with SARS-COV-2 at around day 15 after the first case of SARS-COV-2 infection was reported. The total deaths of individual infected with SARS-COV-2 also increased exponentially at around day 30 after the first case of SARS-COV-2 infection became known. However, while the total number of individuals infected with SARS-COV-2 continued an upward trend, the number of deaths of individuals infected with SARS-COV-2 appeared to stabilize at around 255 deaths per 10,000 individuals infected with SARS-COV-2 (or DP10000 Number of 255). It is not clear whether this was due to successful mitigation of the effects of SARS-COV-2 infections or to factors inherent in the biochemistry (or molecular biology) of SARS-COV-2.

The number of deaths per 10,000 individuals infected with SARS-COV-2 (DP10000 Number) was calculated for different populations in different parts of the world. Figure 2 shows that the deaths per 10,000 individuals infected with SAS-COV-2 (DP10000 Number) varied quite considerably in different populations and regions of the world 
(Figure 2). The lowest value (118 deaths per 10000 individuals infected with SARSCOV-2 or DP10000 Number of 118) was obtained for Germany while the highest value (1190 deaths per 10000 individuals infected with SARS-COV-2 or DP10000 Number of 1190), representing an increase of almost $1000 \%$ was obtained for Italy. The DP10000 Number for the United States was 196 (or 196 Deaths Per 10000 individuals infected with SARS-COV-2) which came to about $170 \%$ of Germany's value. Although the United States had the highest number of individuals infected with SARS-COV-2, it did not have the highest DP10000 Number (or Deaths Per 10000 individuals infected with SARS-COV-2). These data suggest that the Medical Infrastructure, and Response Strategy and Protocol to SARS-COV-2 infections in Germany were better suited to mitigate the effects of SARS-COV-2 on morbidity and that despite the high level of the number of individuals infected with SARS-COV-2, the confusion and the incompetence of the leaderships of the National Institutes of Health (NIH), National Institute of Allergy and Center for Disease Control and Prevention (CDC) in the United States, the Healthcare Professional, including the Physicians, Nurses and Paramedical Personnel have been able to mitigate somewhat the Viral Pandemic due to SARS-COV-2 in the United States. It is anticipated that as the testing for SARS-COV-2 in the general population increases, the DP10000 Number (Deaths per 10,000 Individuals infected with SARS-COV-2 will be decreased even further. Like Germany, Turkey and Switzerland are two countries that appeared to have been able to mitigate COVID-19. The country of Iran had a DP Number of 626 representing almost 530\% above that of Germany. One can only speculate about the reason for the high DP10000 Number for Iran. It is possible that Iran does not have a very sophisticated Medical Infrastructure and the resource to deal with a sudden Viral Pandemic.

However, it is difficult to explain why countries including Italy, Spain, France, Netherlands, Belgium, all members of the European Union with very similar Healthcare System and fairly sophisticated Medical Infrastructure that is comparable to that of Germany and relatively high degree of competence of their Healthcare Professionals, were not able to counteract the effects SARS-COV-2 with respect to their DP10000 Numbers (or Deaths Per 100000 Individuals Infected with SARS-COV-2 . The DP10000 
Numbers for Italy, Spain, Netherlands Belgium, and France were 1190, 904, 861, 659 and 285 respectively representing $\sim 1000 \%, 766 \%, 730 \%, 559 \%$ and $242 \%$ of that of Germany. Incompetence of the leaderships of their Medical Research and Response Agencies may partly explain these abominable DP10000 Numbers but it is submitted that there may be explanations that have to with SARS-COV-2 itself, in particular the biochemistry (or molecular biology) of SARS-COV-2 due to mutational events that may have affected and changed its Virulence.

There has not been any studies of the Virulence of SARS-COV-2 in different populations and environments. Such studies are greatly anticipated. However, current available data points to differential Virulence of SARS-COV-2 in different populations and environments. Figure 3 shows that there was no correlation between Total Number of Individuals Infected with SARS-COV-2 in different populations and environments and Total Number of Deaths or DP10000 Number (Deaths Per 10000 individuals infected with SARS-COV-2. These results strongly suggest that there may be different SARSCOV-2 strains within different populations and environments.

The sequences of several SARS-COV-2 strains have been determined [5]. Lu et al. [2] reported that they sequenced SARS-COV-2 genomes from nine individuals from the same location and found that they had 99.98 sequence identity. However, analysis of the sequences of SARS-COV-2 in different populations and environments must be made to determine differences in Virulence that are populations and environments specific. Pursuant to the Theory of Chaos [6], small but significant changes can in principle have tremendous impact that can lead to Irreversible and Complete Chaos in the form of death as seen in SARS-COV-2 infections. The primary structures of Protein ORF8 encoded by the genomes of SARS-COV-2 strains from various regions were compared. They showed that there were noticeable mutations in various populations and regions of the world (Figure 4). There was also amino acid substitutions in the Spike Protein and Nucleocapsid Phosphoprotein (Figures 5 and 6). These results showed that there were small but significant differences between SARS-COV-2 strains from China and Europe and the United States. 
There is no definitive data that pinpoints the origin of SARS-COV-2. It has been suggested that SARS-COV-2 may originate from bats [1,2,7-9]. However, SARS-COV like viruses are also harbored by other mammals and reptiles, including turtles and snakes $[7,8]$. Irrespective of the origin of SARS-COV-2, it is clear that the SARS-COV-2 strains that infect humans in various countries have undergone some mutations and are most probably going to continue to mutate leading to enhanced or decreased Pathogenicity and Virulence. There is no correlation between Total Number of Individuals Infected with SARS-COV-2 and Total Number of Deaths due to SARS-COV-2 Infects or DP10000 Numbers (Deaths Per 10000 Individuals Infected with SARS-COV-2) (Figure 3). Medical Infrastructure, Preparedness and Response Strategy alone cannot explain the lack of correlation between Total Number of Individuals Infected with SARS-COV-2 and DP10000 Numbers (or Deaths Per 10000 Individuals Infected with SARS-COV-2). It is hypothesized and submitted that correlation between mutations in SARS-COV-2 from different regions and Pathogecity and Virulence could explain the differential DP10000 Numbers (Death Per 10000 Individuals Infected with SARS-COV-2) in different populations and regions of the world. Thus, to mitigate mortality due to SARS-COV-2 infections such as the development of Vaccines and Prophylactics that target SARSCOV-2 encoded proteins, it is necessary to identify mutations within the genomes of SARS-COV-2 strains that enhance its Pathogenecity and Virulence. There is some evidence indicating that there are small but significant changes in the primary structures of proteins encoded by the genomes of various SARS-COV-2 strains, including the Spike Protein (Figure 4) the Orf8 Protein (Figure 5) and the Nucleocapsid Phosphoprotein (Figure 6). The consequences of the mutations in the Spike Protein and Orf8 Protein described in Figures 4 and 5 are presently unknown. The mutation of serine 198 to leucine of the Nucleocapsid Phosphoprotein is of major consequence because serine 197 is a potential phosphorylation site for Aurora A and B kinases which are involved in the control of the cell cycle and proper chromosone segregation [9-11]. The mutation of phospho-serine 197 would also have major ramification for the adjacent threonine 198 which is a potential phosphorylation site for the proline-directed protein kinases, including GSK-3 and Cdk-1 [12-16]. The destruction of phospho-serine 197 
prevents the regulation of phosphorylation of threonine 198 by GSK-3 [Tung, H.Y.L., Manuscript in preparation]. The mutations of Arginine 203 and Glycine 204 of the Nucleocapsid Phosphoprotein will also affect the regulation of the phosphoryltions of serines 202 and 206 which are phosphorylation sites for GSK-3 and CDK-1 respectively [12-16]. Tang et al. [17] suggested that there may be two SAR-COV-2 subtypes and that one subtype termed L Subtype may be more "agressive" in either infectivity (pathogenicity) or virulence than an ancestral subtype termed S Subtype. Ou et al. [18] reported that the protein sequences of the Receptor Binding Domain (RBD) of the Spike Protein of SARS-COV-2 which binds to ACE2 and is necessary for viral entry were susceptible to mutations, and that the Equilibrium Dissociation Constant $\left(\mathrm{K}_{\mathrm{d}}\right)$ of three of these mutants that were associated with Wuhan and Shenzhen, China, and France had $\mathrm{K}_{\mathrm{d}}$ that were two orders of magnitude lower than the prototype Wuhan-Hu 1 strain. Mutations in proteins encoded by the SARS-COV-2 genomes can be accompanied by decreased or enhances Infectivity or Pathogenecity and Virulence. Detailed analysis of mutations associated with increased infectivity or pathogenecity and virulence is necessary for the development of an effective vaccine or prophylactic as it would be disaster if the developed vaccine or prophylactic targets the strain that is associated with decreased infectivity or pathogenecity and virulence.

\section{References.}

1. Zhu, N., Zhang, D., Wang, W., Li, X., Yang, B., Song, J., Zhao, X., Huang, B., Shi, W., Lu, R., Niu, P., Zhan, F., Ma, X., Wang, D., Xu, W., Wu, G., Gao, G.F. and Tan, W. (2019) N. Engl. J. Med., Vol. 382, pp727-733.

A novel coronavirus from patients with pneumonia in China, 2019.

2. Lu, R., Zhao, X., Li, J., Niu, P., Yang, B., Wu, H., Wang, W., Song, H., Huang, B., Zhu, N., Bi, Y., Ma, X., Zhan, F., Wang, L., Hu, T., Zhou, H., Hu, Z., Zhou, W., Zhao, L., Chen, J., Meng, Y., Wang, J., Lin, Y., Yuan, J., Xie, Z., Ma, J., Liu, W.J., Wang, D., Xu, W., Holmes, E.C., Gao, G.F., Wu, G., Chen, W., Shi, W. and Tan, W. (2020) Lancet, Vol. 395, pp565-574. 
Genomic characterisation and epidemiology of 2019 novel coronavirus: implications for virus origins and receptor binding.

3. Zhou, P., Yang, X.L., Wang, X.G., Hu, B., Zhang, L., Zhang, W., Si, H.R., Zhu, Y., Li, B., Huang, C.L., Chen, H.D., Chen, J., Luo, Y., Guo, H., Jiang, R.D., Liu, M.Q., Zhao, K., Chen, Q.J., Deng, F., Liu, L.L., Yan, B., Zhan, Q.J., Deng, F., Liu, L.L., Yan, B., Zhan, F.X., Wang, Y.Y., Gao, G.F. and Shi, Z.L. (2020) Nature, Vol. 579, pp270-273.

A pneumonia outbreak associated with a new coronavirus of probable bat origin.

4. Wu, F., Zhao, S., Yu, B., Chen, Y.-M., Wang, W., Song, Z.-G, Hu, Y., Tao, Z.W., Tian, J.-H., Pei, Y.-Y., Yuan, M.-L., Zhang, Y.-L., Dai, F.-H., Liu, Y., Wang, Q.-M., Zheng, J.-J., Xu, L., Holmes, E.C. and Zhang, Y.Z. (2020) Nature, Vol., 579, pp265-269.

A new coronavirus associated with human respiratory diseaase in China.

5. ncbi.nml.nih.gov/genbank/sars-cove2-seqs/

6. Tung, H.Y.L. (2020) A new theory of chaos, an introduction and its application in the study of climate change, diabetes, life and death, and SARS-COV-2, Cactoa Scientific Publishers, Inc., Astoria (NYC), New York, U.S.A.

7. Liu, Z., Xiao, X., Wei, X., Li, J., Yang, J., Tan, H., Zhu, J., Zhang, Q., Wu, J. and Liu, L. (2020) J. Med. Virol., DOI: 10.1002/jmv.

Composition and divergence of coronovirus spike proteins and host ACE2 receptors predict potential intermediate hosts of SARS-CoV-2.

8. Wei, J., Wang, W., Zhao, X., Zai, J. and Li, X. (2020) J. Med. Virol., DOI: 10.1002/jmv.25682.

Cross-species transmission of the newly identified coronavirus 2019-nCov.

9. Francisco, L., Wang, W., \& Chan, C. S. (1994) Mol. Cell. Biol., Vol. 14, pp47314740.

Type 1 protein phosphatase acts in opposition to IpL1 protein kinase in regulating yeast chromosome segregation.

10. Tung, H.Y.L, Wang, W., Chan, C. S. (1995) Mol. Cell. Biol., Vol. 15, pp6064-6074. 
Regulation of chromosome segregation by Glc8p, a structural homolog of mammalian inhibitor 2 that functions as both an activator and an inhibitor of yeast protein phosphatase 1.

11. Fu, J., Bian, M., Jiang, Q. and Zhang, C. (2007) Mol. Can. Res., Vol. 5, pp1-10. Roles of Aurora Kinases in Mitosis and Tumorigenesis.

12. Fiol, C.J., Mahrenholz, A.M., Wang, Y., Roeske, R.W. and Roach, P.J. (1987) J. Biol. Chem., Vol. 262, pp14042-14048.

Formation of protein kinase recognition sites by covalent modification of the substrate. Molecular mechanism for the synergistic action of casein kinase II and glycogen synthase kinase 3 .

13. Frame, S., \& Cohen, P. (2001) Biochem. J., Vol. 359, pp1-16.

GSK3 takes centre stage more than 20 years after its discovery.

14. Kaidanovich-Beilin, O., \& Woodgett, J. R. (2011). Frontiers in Molecular Neuroscience.

GSK-3: Functional Insights from Cell Biology and Animal Models.

15. Holt, L. J., Tuch, B. B., Villén, J., Johnson, A. D., Gygi, S. P., and Morgan, D. O. (2009). Science, Vol. 325, pp1682-1686.

Global analysis of Cdk1 substrate phosphorylation sites provides insights into evolution.

16. Songyang, Z., Blechner, S., Hoagland, N., Hoekstra, M.F. and PiwnicaWorms, H. and Cantley, L.C. (1994) Curr. Biol. Vol. 4, pp973-982.

Use of an oriented peptide library to determine the optimal substrates of protein kinases.

17. Tang, X., Wu, C., Li, X., Song, Y., Yao, X., Wu, X., Duan, Y., Zhang, H., Wang, Y., Qian, Z., Gui, J. and lu, J. (2020) National Science Review, https:// doi.or/ 10.1093/nsr/nmaa0036/5775463.

On the origin and continuing evolution of SARS-CoV-2.

18. Ou, J., Zhou, Z., Zhang, J., Lan, W., Zhao, Wu, J., Seto, D., Zhang, G. and Zhang, Q. (2020) bioRxiv, https://doi.org/10.1101/2020.03.15.991844.

RBD mutations from circulating SARS-CoV-2 strains enhance the structure stability and infectivity of the spike proteins. 


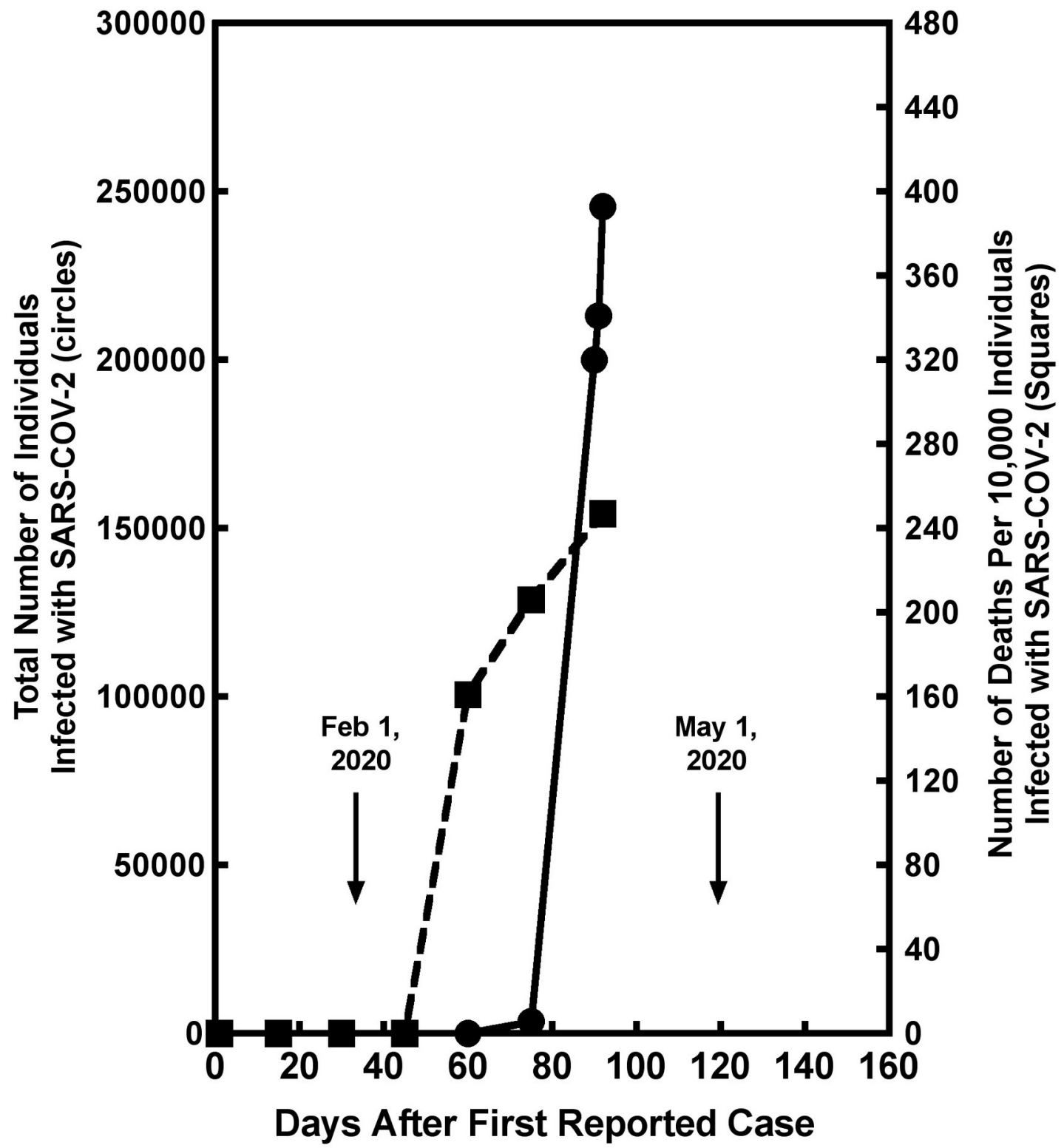

Figure 1. Graph of Total Number of Individuals Infected with SARS-COV-2 in the United States and Number of Deaths Per Ten Thousands Individuals Infected with SARS-COV-2 against Days After First Reported Case of SARS-COV-2 Infection. The data is for the United States up to April 1, 2020. 


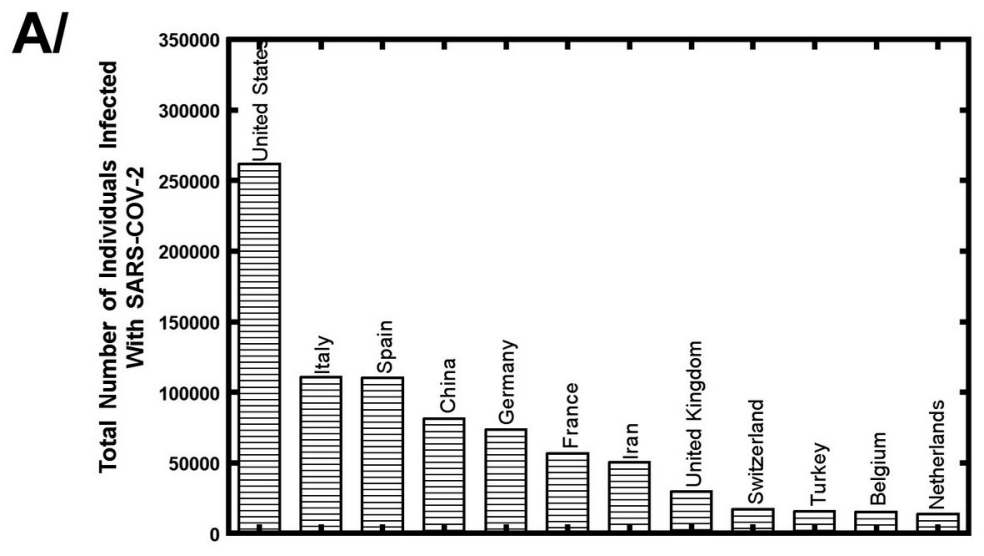

B/
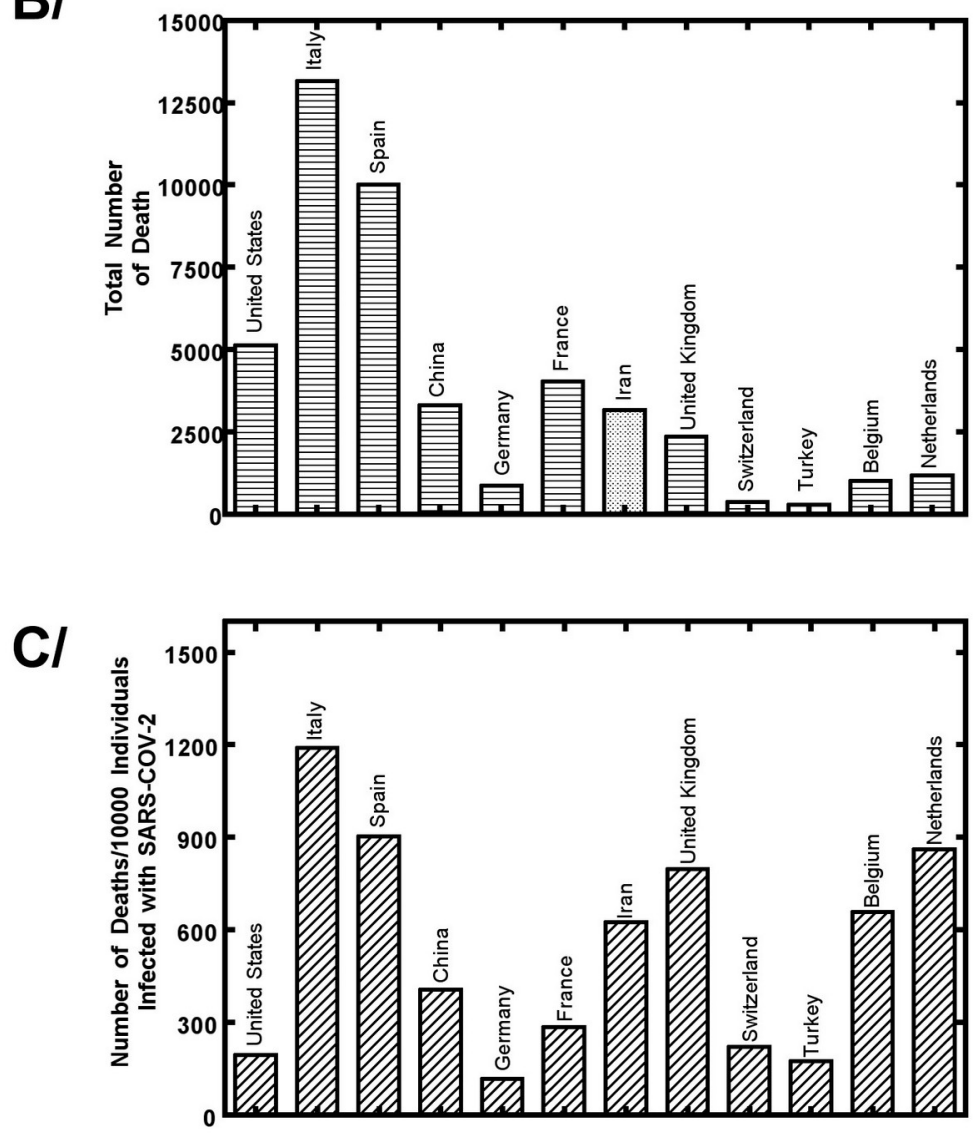

Figure 2. Comparision of the Total Number of Individuals Infected with SARSCOV-2 (Panel A), Total Number of Deaths due to SARS-COV-2 (Panel B), and Number of Deaths Per 10000 Individuals Infected with SARS-COV-2 (Panel C) in different countries, including the United States, Italy, Spain, China, Germany, France, Iran, United Kingdom, Switzerland, Turkey, Belgium and Netherlands. The data is for the United States on March 31, 2020. 


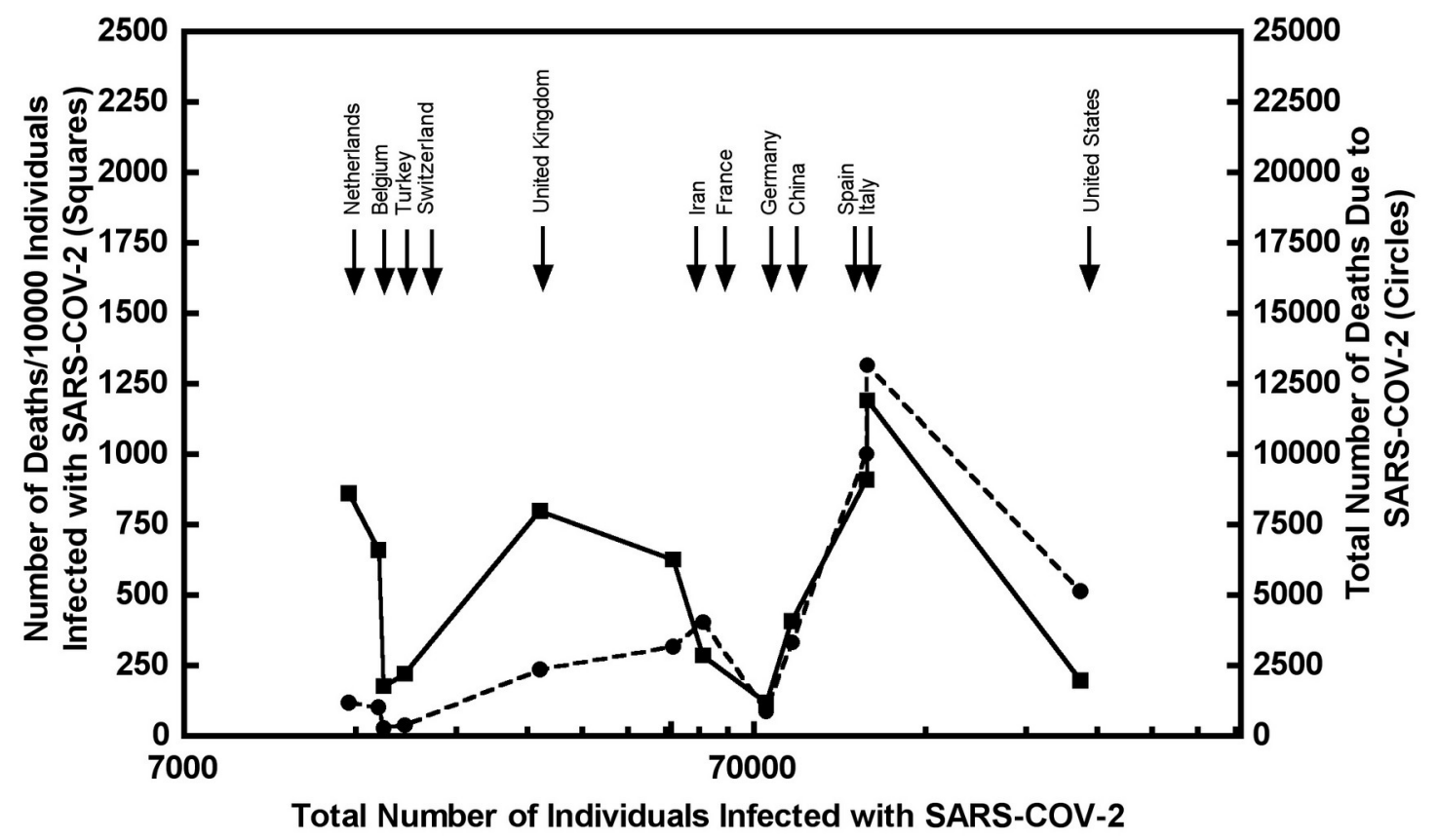

Figure 3. No Correlation between Total Number of Individuals Infected with SARSCOV-2 and Total Number of Deaths due to SARS-COV-2 and Number of Deaths Per 10000 Individuals Infected with SARS-COV-2 (DP10000) The data is for the United States on March 31, 2020. 


\begin{tabular}{|c|c|}
\hline China 1: & 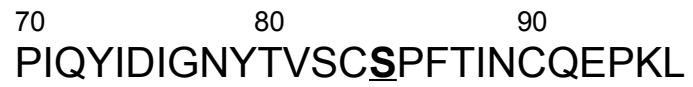 \\
\hline China 2: & PIQYIDIGNYTVSCSPPFTINCQEPKL \\
\hline China 3: & PIQYIDIGNYTVSSLPFTINCQEPKL \\
\hline Italy: & PIQYIDIGNYTVSCLPFTINCQEOKL \\
\hline Spain & PIQYIDIGNYTVSCLPFTINCQEPKL \\
\hline Sweden: & PIQYIDIGNYTVSCLPFTINCQEPKL \\
\hline Finland: & PIQYIDIGNYTVSCLPFTINCQEPKL \\
\hline United States: & PIQYIDIGNYTVSCLPFTINCQEPKL \\
\hline Israel: & PIQYIDIGNYTVSCLPFTINCQEPKL \\
\hline
\end{tabular}

Figure 4. Protein Sequence Alignment of SARS-COV-2 encoded protein, ORF8 (between amino acids 70 and 95) from different coountries including China, Italy, Spain, Sweden, Finland, United States and Israel. The Protein Sequences were downloaded from NCB1. 
A.

China 1:

Finland:

\section{YYPDKVFRSSVLHSSTQDLFLPFFS}

YYPDKVFRSSVLYYSTQDLFLPFFS

B.

$\begin{array}{lc} & 520 \\ \text { China 1: } & 530 \\ \text { Spain: } & \text { FELLHAPATVCGPKKSTNLVKNKCVV } \\ & \end{array}$

C.

China 1:

$601 \quad 610 \quad 620$

GTNTSNQVAVLYQDVVNCTEVPVAI

USA:

GTNTSNQVAVLYQG VNCTEVPVAI

D. 
China 1:

$601 \quad 610$

Israel: $\quad$ GTNTSNQVAVLYQGVNCTEVPVAI

E.

China 1:

$790 \quad 800 \quad 810$

IYKTPPIKDFGGFNFSQILPDPSK

Sweden:

IYKTPPIKDㅌGGFNFSQILPDPSK

Figure 5. Protein Sequence Alignment of SARS-COV-2 encoded Spike Protein, from different coountries including $A$, China and Finland (between amino acids 3660); B, China and Spain, (between amino acids 515-540); C, China and United States, (between amino acids 601-624); D, China and Israel (between amino acids 601-624), E; China and Sweden (between amino acids 790-812). 


\begin{tabular}{|c|c|}
\hline & 200 \\
\hline China 1: & SQASSRSSSRSRNSSRNSTPGSSRGTSPARM \\
\hline Spain: & SQASSRSSSRSRNSSRNLTPGSSRGTSPARM \\
\hline Israel: & 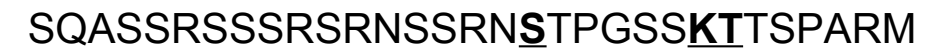 \\
\hline
\end{tabular}

Figure 6. Protein Sequence Alignment of SARS-COV-2 encoded Nucleocapsid Phosphoprotein, from different coountries including China, Spain and Israel (between amino acids 80-110); B, China and Israel, (between amino acids 180-210). 